\title{
A mutation in the Drosophila melanogaster eve stripe 2 minimal enhancer is buffered by flanking sequences
}

Francheska Lopez-Rivera*, †, 1 , Olivia K. Foster*, Ben J. Vincent, 2, Edward C. G. Pym*, Meghan D. J. Bragdon*, 3 , Javier Estrada*, 4 , Angela H. DePace*, Zeba Wunderlich*, 5

*: Department of Systems Biology, Harvard Medical School, Boston, MA 02115

†: GSAS Research Scholar Initiative, Harvard University, Cambridge, MA 02138

1: Current address: Department of Genetics, Harvard Medical School, Boston, MA 02115

2: Current address: Department of Biological Sciences, University of Pittsburgh, Pittsburgh, PA 15260

3: Current address: Department of Biomedical Engineering, Boston University, Boston, MA 02215

4: Current address: Novartis Institutes for Biomedical Research, Cambridge, MA 02139 5: Department of Developmental and Cell Biology, University of California, Irvine, Irvine, CA 92697 
bioRxiv preprint doi: https://doi.org/10.1101/2020.06.19.162164; this version posted June 20,2020. The copyright holder for this preprint (which was not certified by peer review) is the author/funder, who has granted bioRxiv a license to display the preprint in perpetuity. It is made available under aCC-BY-NC 4.0 International license.

Running title (35 char, including spaces) Buffering enhancer mutations

Key words: Drosophila melanogaster, enhancer, even-skipped, transcription factor binding site

Corresponding author:

Zeba Wunderlich

4107 Natural Sciences 2

Irvine, CA 92697

949-824-5959

zeba@uci.edu 


\section{Abstract}

Enhancers are DNA sequences composed of transcription factor binding sites that drive complex patterns of gene expression in space and time. Until recently, studying enhancers in their genomic context was technically challenging. Therefore, minimal enhancers, the shortest pieces of DNA that can drive an expression pattern that resembles a gene's endogenous pattern, are often used to study features of enhancer function. However, evidence suggests that some enhancers require sequences outside the minimal enhancer to maintain function under environmental perturbations. We hypothesized that these additional sequences also prevent misexpression caused by a transcription factor binding site mutation within a minimal enhancer. Using the Drosophila melanogaster even-skipped stripe 2 enhancer as a case study, we tested the effect of a Giant binding site mutation (gt-2) on the expression patterns driven by minimal and extended enhancer reporter constructs. We found that, in contrast to the misexpression caused by the gt-2 binding site mutation in the minimal enhancer, the same gt-2 binding site mutation in the extended enhancer did not have an effect on expression. The buffering of expression levels, but not expression pattern, is partially explained by an additional Giant binding site outside the minimal enhancer. Mutating the gt-2 binding site in the endogenous locus had no significant effect on stripe 2 expression. Our results indicate that rules derived from mutating enhancer reporter constructs may not represent what occurs in the endogenous context. 


\section{Introduction}

Many developmental genes are expressed in complex patterns in space and time. The instructions for these patterns are largely encoded in enhancers, stretches of DNA composed of transcription factor (TF) binding sites. The earliest studies of enhancer function established that enhancers can retain their activity in synthetic reporter constructs, giving rise to the widely-held notion that enhancers are modules with distinct boundaries (Shlyueva et al. 2014). The idea that enhancers have distinct boundaries is reinforced by the way enhancers were traditionally identified - by reducing the DNA upstream of a gene's promoter into increasingly small fragments until a "minimal" enhancer that was sufficient to produce all or a subset of a gene's expression pattern was identified. Even when using modern functional genomic methods, enhancers are annotated with finite boundaries and attempts are often made to identify the minimal enhancer (Arnold et al. 2013; Koenecke et al. 2016; Diao et al. 2017; Monti et al. 2017).

Minimal enhancer reporter constructs have been a powerful tool for studying transcriptional control. By mutating minimal enhancers in reporters, scientists have identified key roles for transcription factor (TF) binding sites (Ney et al. 1990; Arnosti et al. 1996; Ma et al. 2000; Milewski et al. 2004). With the advent of high-throughput DNA synthesis and sequencing, this approach has been extended to study the effects of large numbers of enhancer variants in massively parallel reporter assays (Patwardhan et al. 2009; Melnikov et al. 2012; Inoue and Ahituv 2015; White 2015). An important, but often unstated assumption of this approach is: to decipher regulatory genetic variation in the intact genome, we can extrapolate from the measurements of variation in reporters 
driven by minimal enhancers, if we assume that enhancers are modular; in other words mutations would behave identically in an isolated enhancer and in the genome. Here, we set out to test this assumption directly.

There are several observations that enhancer function, particularly as defined by a minimal enhancer, may not be completely modular (Spitz and Furlong 2012; Lim et al. 2018). When measured quantitatively, the expression driven by some enhancer reporters does not precisely match the endogenous pattern (Staller et al. 2015). In many loci, the paradigm of a single enhancer driving expression in a single tissue is often an oversimplification. For example, in some loci, minimal enhancers cannot be identified for a given expression pattern, and many genes are controlled by seemingly redundant shadow enhancers (Barolo 2012; Sabarís et al. 2019). Furthermore, enhancer boundaries defined by DNAse accessibility and histone marks often do not match minimal enhancer boundaries defined by activity in reporters (Kwasnieski et al. 2014; Henriques et al. 2018). In some cases, the minimal enhancer is sufficient for an animal's viability under ideal conditions, but sequences outside of the minimal enhancer are required for viability when the animal is exposed to temperature perturbations (Ludwig et al. 2011). Together, these examples highlight that while minimal enhancer regions can approximate the expression patterns of a gene, sometimes very closely, quantitative measurements of these regions' activities can reveal their inability to recapitulate the nuances of gene regulation in the endogenous context.

In this work, we directly test the assumption that the misxpression caused by a mutation in a minimal enhancer reporter construct will also be observed when the same mutation is found in the genome. We compared the changes in gene expression caused 
by a mutation in three versions of an enhancer: 1) a minimal enhancer in a reporter, 2) an extended enhancer that contains the minimal enhancer plus flanking sequences in a reporter, and 3) in the endogenous locus. If the minimal enhancer truly represents a modular functional enhancer unit, the effects of the mutation on gene expression will be the same in each of these contexts. If not, the effects caused by the mutation will differ.

We use the well-studied Drosophila melanogaster even-skipped (eve) stripe 2 enhancer as our case study for several reasons (Goto et al. 1989; Small et al. 1992). Eve encodes a homeodomain transcription factor essential for proper segment formation in Drosophila, and five well-characterized enhancers drive its seven-stripe expression pattern in the blastoderm embryo (Figure 1A). To understand the mechanism of eve stripe 2 enhancer function, classic experiments mutated transcription factor binding sites in minimal enhancer reporter constructs, resulting in a set of variants with known effects that we can test in an extended enhancer construct and in the endogenous locus (Small et al. 1992; Arnosti et al. 1996). Subsequent experiments showed that, while the eve stripe 2 minimal enhancer is sufficient for an animal's viability in $D$. melanogaster, the sequences outside of minimal enhancer are required to drive robust patterns of gene expression when the animal is exposed to temperature perturbations (Ludwig et al. 2011), or to drive a proper stripe in other species (Crocker and Stern 2017). Together, these experiments indicate that the minimal enhancer does not recapitulate the complete transcriptional control of eve stripe 2. The Drosophila blastoderm embryo also provides technical advantages; we can readily incorporate reporter constructs, make genomic mutations, and measure levels and patterns of gene expression at cellular resolution (Hendriks et al. 2006; Wunderlich et al. 2014). This 
allows us to measure potentially subtle differences in expression patterns and levels driven by different enhancer variants.

We hypothesized that a transcription factor binding site mutation will have its maximum effect on gene expression when found in a minimal enhancer, while its effects will be reduced, or buffered, when found in the extended enhancer and in the endogenous locus due to the contributions of additional regulatory DNA sequences. We tested our hypothesis and found that the effects of a TF binding site mutation on gene expression are indeed buffered in the extended eve stripe 2 enhancer and in the endogenous locus. This buffering is partially explained by an additional binding site in the sequence outside the eve stripe 2 minimal enhancer. These results imply that we cannot always extrapolate the effects of enhancer mutations in minimal reporters to extended sequences or to the endogenous intact locus. We discuss implications of our results for studying the functional consequences of regulatory sequence variation.

\section{Materials and Methods}

\section{Enhancer sequences and mutations in reporter constructs}

Each of the eve stripe 2 enhancer sequences was cloned into a $\mathrm{pB} \phi \mathrm{Y}$ plasmid containing an eve basal promoter-lacZ fusion gene, the mini-white marker, and an attB integration site. The enhancer sequences are located immediately upstream of the eve basal promoter. All constructs were integrated by Genetic Services, Inc. into the attP2 docking site of the Drosophila melanogaster $y[1], w[67 c 23]$ line. We followed the miniwhite eye marker as we conducted crosses to make the transgenic fly lines homozygous. 
The 484 base pair (bp) wild-type minimal (minWT) enhancer sequence was defined by Small and colleagues (Small et al. 1992). Min $\Delta$ gt is the minWT enhancer with a 43 bp deletion of the giant-2 (gt-2) binding site as described in (Small et al. 1992). The wild-type extended (extWT) enhancer is the minWT sequence plus the 50 bp upstream and 264 bp downstream flanking sequences present in the eve locus. The boundaries of the extWT enhancer are two conserved blocks of 18 and 26 bp on the 3' and 5' ends of the enhancer (Ludwig et al. 1998). The ext $\Delta$ gt enhancer consists of the extWT enhancer with the same gt-2 binding site deletion as in $\min \Delta \mathrm{gt}$.

To computationally predict additional Gt sites in the extended enhancer, we used PATSER and three different Gt position weight matrices (PWMs) generated with data from yeast one-hybrid, DNA footprinting, and SELEX assays (Hertz and Stormo 1999; Noyes et al. 2008; Li et al. 2011; Schroeder et al. 2011). A common Gt binding site was found in the downstream flanking sequence of the extended enhancer using all three PWMs with a p-value of 0.001 . Because of overlaps with other predicted binding sites, this $\mathrm{Gt}$ binding site was mutated by changing five nucleotides in ext $\Delta$ gt to create the ext $\Delta g t, \Delta g t$ enhancer.

The minWT-sp1 and minWT-sp2 enhancers consist of the minWT enhancer and two different 264 bp downstream spacer sequences, sp1 and sp2. Each of these sequences are about half of a $500 \mathrm{bp}$ lacZ sequence from which we removed high affinity binding sites for Bicoid, Hunchback, Giant, and Kruppel, using a PATSER p-value of 0.003. The $\min \Delta \mathrm{gt}-\mathrm{sp} 1$ enhancer is composed of $\min \Delta \mathrm{gt}$ and $\mathrm{sp} 1$. Min $\Delta \mathrm{gt}-\mathrm{sp} 1+\mathrm{gt}$ is the $\min \Delta$ gt-sp1 enhancer containing the additional $g t$ binding site that we identified, located 
in the position where it is found in the extended enhancer. File S1 contains the sequences of all the enhancers that were tested in reporter constructs.

Endogenous eve giant-2 deletion using the CRISPR system

Briefly, gRNAs (5'-TCTAACTCGAAAGTGAAACGAGG-3' and 5'-

ATTCCGTCTAAATGAAAGTATGG-3') adjacent to the gt-2 binding site were cloned into pU6-Bbsl-chiRNA. A ScarlessDsRed selection cassette (https://flycrispr.org/scarlessgene-editing/) was used with $\sim 500$ bp homology arms flanking the gRNA cut sites in the eve stripe 2 enhancer. These plasmids were injected into $y[1] w[67 c 23]$; attP2\{nosCas9\} by BestGene. The dsRed selection cassette was mobilised by crossing to w[1118]; In(2LR)Gla, wg[Gla-1]/CyO; Herm\{3xP3-ECFP,alphatub-piggyBacK10\}M10, and selecting for non-dsRed eyed flies, to give the final allele eve[ahd4]. Further crosses to remove the transposase yielded flies with the genotype $w$ [1118]; eve[ahd4], which we term " $\Delta$ gt eve locus." The edit was confirmed by PCR. The control flies to which the CRISPR flies were compared had the genotype $y[1] w[67 c 23]$; attP2 $\{\mathrm{hbP} 2-$ LacZ\}.

In situ hybridization and imaging

We collected and fixed 0-4 hour old embryos grown at $25^{\circ} \mathrm{C}$, and we stained them using in situ hybridization as in (Hendriks et al. 2006; Wunderlich et al. 2014). We incubated the embryos at $56^{\circ} \mathrm{C}$ for two days with DNP-labelled probes for $h k b$ and DIGlabelled probes for ftz. Transgenic reporter embryos were also incubated with a DNPlabeled probe for lacZ, and the WT eve locus and $\Delta$ gt eve locus CRISPR embryos were 
incubated with a DNP-labeled probe for eve. Hkb probes were used to normalize lacZ expression levels between the different transgenic reporter lines. The DIG probes were detected with anti-DIG-HRP antibody (Roche, Indianapolis, IN) and a coumarintyramide color reaction (Perkin-EImer, Waltham, MA), and the DNP probes were detected afterwards with anti-DNP-HRP (Perkin-Elmer) antibody and a Cy3-tyramide color reaction (Perkin-Elmer). Embryos were treated with RNAse and nuclei were stained with Sytox green. We mounted the embryos in DePex (Electron Microscopy Sciences, Hatfield, PA), using a bridge of \#1 slide coverslips to avoid embryo morphology disruption.

Reporter embryos from the early blastoderm stage (4-10\% membrane invagination, roughly 10-20 minutes after the start of the blastoderm stage) were imaged, and CRISPR embryos from early blastoderm stage (9-15\% membrane invagination, roughly $15-25$ min after the start of the blastoderm stage) were imaged. We used 2-photon laser scanning microscopy to obtain z-stacks of each embryo on a LSM 710 with a plan-apochromat 20X 0.8 NA objective. Each stack was converted into a PointCloud, a text file that includes the location and levels of gene expression for each nucleus (Hendriks et al. 2006).

\section{Data analysis of reporter constructs}

To normalize the lacZ levels in the reporter embryos, we divided the lacZ signal by the $95 \%$ quantile of $h k b$ expression in the posterior $10 \%$ of each embryo (Wunderlich et al. 2014). We expect the lacZ and $h k b$ levels to be correlated within a transgenic line. To verify this, we ran a regression of the $99 \%$ quantile lacZ value from each embryo and 
the $95 \%$ quantile $h k b$ value. Cook's distance (Cook, 1977) was used to discard influential outliers (Wunderlich et al. 2014). To avoid extraneous sources of noise in the normalization, we only compare lacZ levels between embryos with the same genetic background and stained in the same in situ hybridization experiment.

To calculate the average lacZ expression levels along the anterior-posterior (AP) axis in each transgenic line, we used the extractpattern command in the PointCloud toolbox, which can be found in http://bdtnp.lbl.gov/Fly Net/bioimaging.jsp?w=analysis. This command divides the embryo into 16 strips around the dorso-ventral (DV) axis of the embryo, and for each strip, calculates the mean expression level in 100 bins along the anterior-posterior (AP) axis. We averaged the strips along the right and left lateral sides of the embryos and subtracted the minimum value along the axis to remove background noise.

We calculated the peak average lacZ expression level within the eve stripe 2 region for each transgenic line in each in situ experiment separately. We then calculated the ratio between the peak average lacZ expression levels of two transgenic lines stained in the same in situ experiment. Ratios were calculated for each stain and the average ratio from multiple stains was determined. Comparisons between average ratios and 1 or between two different ratios were made by using one- or two-sample ttests with unequal variances.

The boundaries of eve stripe 2 expression were defined as the inflection point of the lacZ expression levels. Since the boundaries of lacZ expression should not change between stains, plots with the average boundaries of lacZ expression in each transgenic line were made with embryos pooled from multiple stains (see Figure S2 for number of 
embryos measured for each genotype). The cell length differences were calculated by determining the average position of the boundary across the DV axis of the embryos analyzed. One cell length is approximately equivalent to one percent of the embryo length.

Data analysis of endogenous eve giant-2 deletion

Briefly, we normalized to eve stripe 1 cellular expression to compare eve levels in the eve[ahd4] embryos and the control (Fowlkes et al. 2008). As described above, using the extractpattern command from the PointCloud toolbox, we found an averaged lateral trace across both sides of the embryo. The peak average eve expression for each stripe was normalized to the peak average expression of eve stripe 1 . We performed a comparison of stripe levels between conditions using a two-sided rank sum test.

The boundary of eve stripes were defined as above using extractpattern and, for a given embryo, eight boundary positions on the left and right lateral sides were averaged. Plots with the average boundary of eve stripe 2 in the eve[ahd4] versus control were made with embryos pooled from different stains. To compare boundaries between the two genotypes, a Mann-Whitney $U$ Test was used, with the factors being one of the eight dorso-ventral positions along both lateral sides of the embryo and the embryo genotype. The p-value was reported for the genotype factor effect.

\section{Data Availability}

All transgenic and CRISPR fly lines are available upon request. File S1 contains the sequences for all enhancer constructs. Figure S2 contains all ratios presented in 
Figures 1-3 in one plot. Figure S5 contains the enhancer sequence of the eve[ahd 4] locus as well as a map of the predicted binding sites.

\section{Results}

The minimal and extended eve stripe 2 enhancers drive different patterns and levels of expression

To test the effects of mutations in the minimal and extended eve stripe 2 enhancer on expression, we began by characterizing the wild type (WT) expression patterns driven by the previously-defined minimal (minWT) and extended (extWT) enhancers (Figure 1B-F). The minimal enhancer is $484 \mathrm{bp}$ and was identified as the smallest piece sufficient to drive expression in the region of stripe 2 (Small et al. 1992). The extended enhancer boundaries were chosen as the two conserved blocks of 18 and 26 bp on the $3^{\prime}$ and 5' sides of the minimal enhancer, resulting in a 798 bp piece (Ludwig et al. 1998). We generated transgenic animals with lacZ reporter constructs inserted into the same location of the genome, and we measured lacZ expression using in situ hybridization and a co-stain for normalization (Wunderlich et al. 2014). The stripe driven by the extended enhancer is wider - its anterior boundary is $\sim 1.6$ cell widths more anterior than that of the minimal enhancer (Figure 1F). In addition, the peak lacZ expression driven by the extWT is 1.45 times higher than the minWT enhancer ( $p$-value ratio $>1$ is 0.0373 ; Figure $1 \mathrm{D}, \mathrm{E})$. 
To test the effect of mutations in the minimal and extended enhancers, we looked to the literature to find a known sequence mutation that had a measurable effect on expression in the minimal enhancer. Previous work identified three footprinted binding sites within the minimal enhancer for the repressor Giant (Gt), which is expressed anterior of eve stripe 2 (Figure 1G). A minimal enhancer with a deletion of one of these binding sites, gt-2, drives higher and broader expression than the WT enhancer (Arnosti et al. 1996). We created reporters with the same deletion of gt-2 in the minimal and extended enhancers (Figure $1 \mathrm{H}$ ) and measured the effect of the deletion on both expression levels and patterns. Consistent with previous results, we found that $\min \Delta \mathrm{gt}$ drives 1.67 times the expression of the minWT enhancer ( $p$-value expression ratio $>1$ is 0.0018 in Figure I, top), and a pattern that is expanded 1.7 cell widths to the anterior (Figure $\mathrm{H}$, top). In contrast, the expression level driven by the ext $\Delta \mathrm{gt}$ enhancer is not significantly different from the extWT enhancer ( $p$-value expression ratio $>1$ is 0.4511 in Figure 1I, bottom), and the pattern is expanded by only 0.9 cell widths (Figure $1 \mathrm{H}$, bottom). The $\min \Delta \mathrm{gt} / \mathrm{minWT}$ expression ratio is also significantly larger than the ext $\Delta g t /$ extWT ratio (p-value=0.0032), indicating that the deletion has a much larger effect on the expression level driven by the minimal enhancer than the extended enhancer. Together, these results indicate that the effect of the gt binding site deletion is buffered in the extended enhancer.

Distance from the promoter reduces expression levels and does not explain buffering

The minimal and extended enhancers differ from one another in the flanking sequences. These flanks may contribute to buffering in two primary ways: 1) the flanks 
may contain TF binding sites or other specific sequence elements, and 2) the flanks increase the distance of the minimal piece from the promoter.

In the minWT constructs the enhancer is $38 \mathrm{bp}$ from the promoter, whereas in the extWT constructs the same minWT sequence is located 302 bp away from the promoter. To test how this change in distance contributes to the differences in expression of the two constructs, we inserted two different 264 bp spacer sequences (sp1 and sp2) into the minWT reporters, to make the constructs minWT-sp1 and minWT-sp2 (Figure 2A). sp1 and sp2 are lacZ sequences from which high affinity binding sites for the regulators involved in eve stripe 2 expression have been removed. For both spacers, increasing the distance of the minWT sequence significantly reduces expression levels ( 0.48 for $s p 1, p=7.189 e-4$, and 0.54 for $s p 2, p=0.0010$, Figure $2 C$ ), while only minimally affecting the AP positioning, with both showing marginal shifts to the posterior in comparison to minWT. The anterior and posterior boundaries of the minWT-sp1 are shifted to the posterior part of the embryo by 1.4 and 1.3 cell lengths, respectively, when compared to minWT (Figure 2B). The anterior and posterior boundaries of minWT-sp2 are shifted to the posterior by 1.0 and 1.1 cell lengths, respectively, when compared to minWT (Figure 2B). These data demonstrate that the level of expression driven by minWT is influenced by enhancer-promoter distance.

To test if promoter-enhancer distance explains the buffering of the gt-2 deletion, we made a construct with the $\min \Delta \mathrm{gt}$ enhancer separated from the promoter by $\mathrm{sp} 1$, $\min \Delta$ gt-sp1, and compared it to minWT-sp1 (Figure 2D). If the distance from the promoter contributes to the buffering effect, the expression ratio of min $\Delta$ gt-sp $1 / \operatorname{minWT-}$ sp1 would be smaller than that of $\min \Delta \mathrm{gt} / \mathrm{minWT}$, and the spatial pattern between 
minWT-sp1 and min $\Delta$ gt-sp1 would be more similar than between minWT and min $\Delta$ gt. In fact, the opposite is true - the ratio is larger ( $p$-value $=0.0025)$ and the spatial pattern is less similar, indicating that the relative distance of the core $484 \mathrm{bp}$ to the promoter does not contribute to the buffering in the extended piece (Figure 2E, F).

An additional Gt binding site in the flanking sequence partially explains the buffering

Since promoter-enhancer distance does not explain the buffering of the extended enhancer, the buffering must be due to differences in the sequence content of the minimal and extended enhancers. We hypothesized that there might be additional gt binding sites in the flanks of the extended enhancer that explain the observed buffering of the gt-2 deletion. We scanned these flanking regions with several existing Gt position weight matrices (PWMs) and found one binding site common to all the PWMs (see Materials and Methods and Figure S1). We mutated the common site to make the ext $\Delta g t, \Delta g t$ construct (Figure 3A). If this common site is responsible for the buffering, we would expect that the ext $\Delta \mathrm{gt}, \Delta \mathrm{gt}$ construct would drive higher expression levels and a wider stripe than the extWT construct. The ext $\Delta \mathrm{gt}, \Delta \mathrm{gt}$ enhancer drives a pattern with an anterior boundary that is not significantly different from the ext $\Delta$ gt enhancer (Figure 3B). Compared to the peak expression levels driven by the extWT enhancer, the ext $\Delta \mathrm{gt}, \Delta \mathrm{gt}$ enhancer drives 1.20 times the expression ( $p$-value that ratio $>1$ is 0.0654 ) (Figure $3 C$ ). Because the peak expression ratio of ext $\Delta \mathrm{gt}, \Delta \mathrm{gt} / \mathrm{extWT}$ is between that of $\min \Delta g t / \operatorname{minWT}$ and ext $\Delta \mathrm{gt} / \mathrm{extWT}$, this result suggests that the additional Gt binding site is partially responsible for buffering the effect of the gt-2 mutation on expression levels (Figure S2). However, since the ext $\Delta \mathrm{gt}$ and ext $\Delta \mathrm{gt}, \Delta \mathrm{gt}$ enhancers drive virtually the 
same expression pattern, this binding site is not responsible for buffering the effect of gt-2 deletion on expression pattern. Therefore, this additional Gt binding site can only partially explain why the extended enhancer can buffer the effect of the gt-2 deletion. Additional Gt binding sites, other TF binding sites, or other functional sequences in the extended enhancer sequence flanks may be responsible for the unexplained buffering (see Discussion).

Adding a Gt binding site to the minimal enhancer is not sufficient to buffer a Gt mutation

Since the additional Gt site is necessary to partially buffer the gt-2 deletion, we wanted to test whether it was also sufficient. We inserted the additional Gt binding site into the spacer of the min $\Delta$ gt-sp1 construct in the same position as it is found in the extWT construct to make the min $\Delta$ gt-sp1+gt construct (Figure 3D). We compared its expression to the minWT-sp1 and the $\min \Delta$ gt-sp1 constructs . If the additional Gt site is sufficient to buffer the gt-2 deletion, we would expect that the min $\Delta$ gt-sp1+gt would drive lower expression levels than min $\Delta$ gt-sp1 and a similar expression pattern to the minWTsp1 construct. We found that the peak expression ratio of $\min \Delta \mathrm{gt}-\mathrm{sp} 1+\mathrm{gt} / \mathrm{minWT-sp} 1$ was on average lower, but not significantly different from the $\min \Delta$ gt-sp $1 / \mathrm{minWT-sp} 1$ ratio, indicating that this binding site alone is not sufficient to buffer the gt-2 deletion ( $p$ value $=0.1729)($ Figure 3F). The expression patterns driven by $\min \Delta$ gt-sp1+gt and $\min \Delta$ gt-sp1 are also very similar, though there is a slight posterior shift of the anterior boundary in the min $\Delta$ gt-sp1+gt construct (Figure 3E). It is possible that this binding site needs its original context to function properly, which may be due to the importance of 
binding site flanks on DNA shape (Rohs et al. 2010; Li and Eisen 2018), or other, unknown requirements.

The gt-2 transcription factor binding site mutation is buffered in the endogenous locus

To test whether the gt- 2 deletion can be buffered in the intact locus, as it is in the extended enhancer, we used CRISPR editing to generate flies homozygous for the same gt-2 deletion in the endogenous eve locus, which we called $\Delta$ gt eve locus (Figure 4A, Figure S5). We then measured eve expression patterns and levels using in situ hybridization in the $\Delta$ gt eve locus embryos and WT eve locus embryos (see Methods for details). To measure expression levels in eve stripe 2, we internally normalized to the levels of eve stripe 1, which is the first eve stripe to be expressed in this developmental stage (Figure S3; see Methods for details). We observed that the expression levels of eve stripe 2 in embryos with $\Delta$ gt eve locus are not significantly different from those in embryos with WT eve locus ( $p$-value $=0.1007)$ (Figure 4C). The eve stripe 2 patterns driven by the WT eve locus and the $\Delta$ gt eve locus are not significantly different (MannWhitney $U$ test, Figure 4B). This suggests that the gt-2 deletion in the endogenous eve stripe 2 enhancer is buffered: expression levels and boundary position in the $\Delta$ gt eve locus embryos are not significantly different from the WT eve locus embryos, in agreement with the observations made in the extended enhancer. Interestingly, we observed differences between $\Delta \mathrm{gt}$ eve locus and WT eve locus on other stripes of the eve pattern (Figure S4). There are differences in the expression levels of eve stripes 5 and 6 , and in the patterns of eve stripe 4 . We speculate that the differences might be due to the effects of the genetic backgrounds of $\Delta$ gt eve and WT eve locus embryos 
(see Methods). All together, these results suggest that the effect of a specific mutation in the eve stripe 2 minimal reporter construct is not recapitulated when tested in the endogenous enhancer context.

\section{Discussion}

The desire to define discrete minimal sequences that are sufficient to drive gene expression patterns emerged from a combination of the technical limitations imposed upon early studies and the resulting "founder fallacy" (Halfon 2019), cementing the first discovered examples of enhancers into generalisations. Understanding and acknowledging the ways in which the activity of minimal enhancers in reporter constructs differs from the activity of the same sequences within the endogenous locus will help us understand gene regulatory logic at a genome scale, as well as regulatory variation and evolution; simultaneously, it reaffirms the important contributions that reporter constructs can still make to deciphering the mechanisms of transcription.

Using one of the textbook examples of an enhancer, eve stripe 2, we have shown that deletion of a key TF binding site for Gt has significant functional effects on the expression driven by the minimal enhancer sequence, but not when this minimal enhancer is modestly extended, nor when the same binding site is removed from the endogenous locus. Furthermore, we identified an additional Gt binding site found outside the minimal enhancer that contributes to buffering the effect of this mutation. However, this additional site partially buffers changes in expression level, but not position, and is necessary, but not sufficient to explain the buffering effect observed in the extended enhancer. 
Given that there were no previously described Gt binding sites in the region flanking the minimal enhancer, it was somewhat unexpected that the effect of the gt-2 deletion would be buffered in the extended enhancer (Ludwig et al. 2011). However, finding all transcription factor binding sites remains a challenge and may explain why we cannot fully account for the gt-2 deletion buffering in the extended enhancer (Keilwagen et al. 2019). Gt's binding preference has been measured using several techniques, which all yield different sequence motifs (Noyes et al. 2008; Li et al. 2011; Schroeder et al. 2011). We searched for Gt binding sites with three different sequence motifs, and we found and mutated a single high-affinity binding site predicted by all three motifs. But, there are additional predicted Gt binding sites that may be contributing to the buffering (Figure S1).

We do not understand why the minimal spacer constructs that include the gt-2 deletion show a large anterior shift of the posterior boundary of the expression pattern. The shift is not observed in the minimal spacer constructs that exclude the deletion or in the $\min \Delta \mathrm{gt}$ or ext $\Delta \mathrm{gt}$ constructs, so it is not due to the spacer sequence or to the gt- 2 mutation individually. We hypothesize there is a specific promoter-enhancer interaction that occurs when both the spacer and the gt-2 deletion are present, but we cannot speculate on the precise underlying cause of this interaction.

This simple case study illustrates clearly that the effects of mutations, as measured in minimal enhancer sequences, cannot be simply extrapolated to larger enhancer regions or to the enhancer in its endogenous context in the genome. These results provide additional evidence challenging the idea that enhancers are strictly modular and that they have defined boundaries (Evans et al. 2012; Halfon 2019; 
Sabarís et al. 2019). Experiments using minimal enhancer reporter constructs have been extremely valuable for identifying genetic interactions and mechanisms of transcriptional control, e.g. activator/repressor balance and short- and long-range repression (Arnosti et al. 1996; Kulkarni and Arnosti 2005; Vincent et al. 2018). However, as more high throughput methods are developed to test the effect of mutations in small to medium-size enhancer fragments, we need to be cautious in interpreting these results (Inoue and Ahituv 2015). A mutation that may have dramatic effects on expression when made in a minimal enhancer may have no effect when made in the genome of an animal.

To test the mutation effects definitively, reporter construct experiments need to be complemented with manipulations of the endogenous enhancer sequences. Due to the CRISPR revolution, these types of experiments are becoming increasingly feasible (Zhou et al. 2014; Kvon et al. 2016; Rogers et al. 2017), and methods are being developed to use high-throughput CRISPR experiments to identify and perturb enhancers, as reviewed in (Lopes et al. 2016; Catarino and Stark 2018). These experiments will provide the data to attack the challenge of modeling the function of increasingly large pieces of the genome simultaneously, which is ultimately required to predict how variation in enhancer sequences affects gene expression. 


\section{Acknowledgements}

We thank all the members of the DePace lab for helpful discussions and suggestions on the manuscript. The research reported in this publication was funded by the Harvard GSAS

Research Scholar Initiative (to F.L.R.), NIH grants K99/R00 HD073191 and R01 HD095246 (to Z.W.), the NSF Graduate Research Fellowship DGE1745303 (to O.K.F.), the Giovanni Armenise-Harvard Foundation's HMS Junior Faculty Grants Program, the Mckenzie Family Charitable Trust Systems Biology Fellowship Fund, the William F. Milton Fund, the Harriet Sugar Toibin Charitable Gift Annuity - Gene Intervention Research, NSF grants 1715184 and IOS-1452557, NIH grants R21 HD072481, R01 GM122928 and U01 GM103804 (to A.H.D.), and the Novartis fellowship (to J.E.). We thank Jeehae Park for her contribution of the fixed y[1] w[67c23]; attP2\{hbP2-LacZ\} embryos used in our analyses. 


\section{Figure Legends}

\section{Figure 1: The effect of the gt-2 binding site mutation is buffered in the eve stripe 2}

extended enhancer. (A) Eve is expressed as a pattern of seven stripes along the anterior-posterior axis of the Drosophila melanogaster blastoderm, and this pattern is driven by five enhancers. (B) We generated transgenic reporter fly lines with the wildtype minimal (minWT) and extended (extWT) eve stripe 2 enhancers, and we measured lacZ expression in embryos using in situ hybridization. (C, D) We plotted lacZ levels in a lateral strip of cells along the AP axis for the minWT (dark gray) and extWT (brown) enhancers measured in a single stain, with the shading showing the standard error of the mean. The extWT enhancer drives a higher peak level of expression. (E) We calculated the ratio of peak lacZ expression levels (black dots in D) driven by the extWT and minWT enhancers in five different stains (open circles). The average ratio of the five stains is represented by a closed circle. The extWT enhancer drives 1.45 times higher expression than the minWT ( $p$-value extWT/minWT > $1=0.0373$ ). $(F)$ We show the average boundary positions of the lacZ expression pattern. Error bars show standard error of the mean boundary positions of the expression pattern. The extWT enhancer drives a wider pattern of expression (brown shading) than the minWT enhancer (gray shading), with the anterior border of the stripe laying $\sim 1.6$ cell widths more anterior than the minWT enhancer pattern. (G) The transcription factor Giant (Gt) is expressed as a broad band anterior to eve stripe 2 and represses eve, establishing the anterior boundary of stripe 2. $(\mathrm{H}, \mathrm{I})$ We characterized the expression patterns and levels driven by the minimal (min $\Delta$ gt, top panels) and extended (ext $\Delta$ gt, bottom panels) enhancers with a gt-2 binding site deletion. In the min $\Delta$ gt enhancer, the gt-2 deletion 
causes an anterior shift in the anterior boundary of the expression pattern and an increase in expression level. In the extended enhancer, the gt-2 deletion causes a more modest shift in the anterior boundary and no significant change in peak expression level.

\section{Figure 2: Distance from the promoter reduces eve stripe 2 expression levels and} is not sufficient to explain the buffering. (A) To test if distance from the promoter contributes to buffering the gt-2 deletion, we used two different 264 bp spacer sequences (sp1 and sp2) to make two constructs, minWT-sp1 and minWT-sp2. (B) We find that moving the minimal enhancer away from the promoter slightly shifts the boundaries of the stripe to the posterior. Error bars show standard error of the mean boundary positions of the expression pattern. (C) A comparison of peak expression levels shows that moving the minimal enhancer away from the promoter reduces peak expression levels. (D) We tested if distance from the promoter is sufficient to explain the Gt site deletion buffering in the extended enhancer by introducing the Gt site deletion into the minWT-sp1 construct, $\min \Delta \mathrm{gt}$-sp1. (E) The min $\Delta \mathrm{gt-sp1}$ construct drives an expression pattern that is dramatically shifted to the anterior, indicating that the spacer cannot buffer the Gt binding site deletion's effect on expression pattern. (F) The min $\Delta$ gtsp1/minWT-sp1 peak expression ratio is significantly larger than $\min \Delta \mathrm{gt} / \mathrm{minWT}$ ratio, indicating that the gt-2 deletion has a more dramatic effect in the min $\Delta$ gt-sp 1 and that the increasing distance from the promoter does not buffer the effects of the gt-2 deletion. 
Figure 3: An additional Gt binding site partially explains the buffering. (A) We found an additional predicted Gt binding site outside the minimal enhancer sequence but within the extended enhancer. A reporter construct, ext $\Delta \mathrm{gt}, \Delta \mathrm{gt}$, testing for the necessity of the additional Gt binding site was made by mutating the predicted Gt binding site. (B) The average position of the lacZ anterior boundaries was nearly identical for the ext $\Delta \mathrm{gt}$ and ext $\Delta \mathrm{gt}, \Delta \mathrm{gt}$ constructs, indicating that eliminating the additional Gt binding site does not affect buffering of the gt-2 deletion of the expression pattern. Error bars show standard error of the mean boundary positions of the expression pattern. (C) If the additional gt site was necessary and sufficient for the buffering, the ext $\Delta \mathrm{gt}, \Delta \mathrm{gt} / \mathrm{extWT}$ ratio would be higher than 1 and very similar to $\min \Delta \mathrm{gt} / \min W T$ ratio. If the additional Gt binding site was not necessary at all, the ext $\Delta g t, \Delta g t / e x t W T$ ratio would be similar to 1 and to the ext $\Delta g t / e x t W T$ ratio. The results suggest that the additional Gt site explains only some of the buffering of the gt-2 deletion in level. (D) We tested the sufficiency of the additional Gt binding site by making a construct with the $\min \Delta \mathrm{gt}$-sp 1 element and inserting the additional Gt binding site in the same location as it is found in the extended enhancer (min $\Delta \mathrm{gt}-\mathrm{sp} 1+\mathrm{gt})$. (E) The additional Gt binding site shifts the anterior boundary of expression slightly to the posterior, when compared to the pattern driven by $\min \Delta \mathrm{gt}-\mathrm{sp} 1$. $(\mathrm{F})$ The peak $\min \Delta \mathrm{gt}-$ $\mathrm{sp} 1+\mathrm{gt} / \mathrm{minWT-sp} 1$ ratio is lower, but not significantly different from the $\min \Delta \mathrm{gt}-$ sp1/minWT-sp1 ratio, indicating that this Gt binding site is not sufficient to explain the buffering of expression level in the extended enhancer. 
Figure 4: CRISPR deletion of Gt binding site in endogenous eve locus does not change stripe 2 expression. (A) Using a scarless-CRISPR method, we removed the gt-2 binding site endogenously. (B) The boundaries of eve stripe 2 are not significantly different between the $\Delta \mathrm{gt}$ eve locus embryos and the WT embryos (Mann-Whitney U test). Error bars show standard error of the mean boundary positions of the expression pattern. This indicates that the boundary of eve stripe 2 in the endogenous context is buffered against the removal of gt-2. (C) Normalized peak expression levels of eve stripe 2 did not change significantly in the $\Delta$ gt eve locus versus control $(p=0.1007)$. Moreover, the ratio of $\Delta \mathrm{gt}$ eve locus to WT eve locus equals 1.1761. Filled circles represent mean expression level and open circles are eve peak expression for each individual embryo analyzed ( $\Delta$ gt locus: $n=11$, control locus: $n=11)$. 


\section{Literature Cited}

Arnold, C. D., D. Gerlach, C. Stelzer, Ł. M. Boryń, M. Rath et al., 2013 Genome-wide quantitative enhancer activity maps identified by STARR-seq. Science 339: 10741077.

Arnosti, D. N., S. Barolo, M. Levine, and S. Small, 1996 The eve stripe 2 enhancer employs multiple modes of transcriptional synergy. Development 122: 205-214.

Barolo, S., 2012 Shadow enhancers: frequently asked questions about distributed cisregulatory information and enhancer redundancy. Bioessays 34: 135-141.

Catarino, R. R., and A. Stark, 2018 Assessing sufficiency and necessity of enhancer activities for gene expression and the mechanisms of transcription activation. Genes Dev. 32: 202-223.

Crocker, J., and D. L. Stern, 2017 Functional regulatory evolution outside of the minimal even-skipped stripe 2 enhancer. Development 144: 3095-3101.

Diao, Y., R. Fang, B. Li, Z. Meng, J. Yu et al., 2017 A tiling-deletion-based genetic screen for cis-regulatory element identification in mammalian cells. Nat. Methods 14: 629-635.

Evans, N. C., C. I. Swanson, and S. Barolo, 2012 Chapter four - Sparkling Insights into Enhancer Structure, Function, and Evolution, pp. 97-120 in Current Topics in Developmental Biology, edited by S. Plaza and F. Payre. Academic Press.

Fowlkes, C. C., C. L. L. Hendriks, S. V. E. Keränen, G. H. Weber, O. Rübel et al., 2008 A quantitative spatiotemporal atlas of gene expression in the Drosophila blastoderm. Cell 133: 364-374. 
Fujioka, M., G. Sun, and J. B. Jaynes, 2013 The Drosophila eve insulator Homie promotes eve expression and protects the adjacent gene from repression by polycomb spreading. PLoS Genet. 9: e1003883.

Goto, T., P. Macdonald, and T. Maniatis, 1989 Early and late periodic patterns of even skipped expression are controlled by distinct regulatory elements that respond to different spatial cues. Cell 57: 413-422.

Halfon, M. S., 2019 Studying Transcriptional Enhancers: The Founder Fallacy, Validation Creep, and Other Biases. Trends Genet. 35: 93-103.

Hendriks, C. L. L., S. V. E. Keränen, C. C. Fowlkes, L. Simirenko, G. H. Weber et al., 2006 Three-dimensional morphology and gene expression in the Drosophila blastoderm at cellular resolution I: data acquisition pipeline. Genome Biol. 7: R123.

Henriques, T., B. S. Scruggs, M. O. Inouye, G. W. Muse, L. H. Williams et al., 2018 Widespread transcriptional pausing and elongation control at enhancers. Genes Dev. 32: 26-41.

Hertz, G. Z., and G. D. Stormo, 1999 Identifying DNA and protein patterns with statistically significant alignments of multiple sequences. Bioinformatics 15: 563577.

Inoue, F., and N. Ahituv, 2015 Decoding enhancers using massively parallel reporter assays. Genomics 106: 159-164.

Keilwagen, J., S. Posch, and J. Grau, 2019 Accurate prediction of cell type-specific transcription factor binding. Genome Biol. 20: 9. 
Koenecke, N., J. Johnston, B. Gaertner, M. Natarajan, and J. Zeitlinger, 2016 Genomewide identification of Drosophila dorso-ventral enhancers by differential histone acetylation analysis. Genome Biol. 17: 196.

Kulkarni, M. M., and D. N. Arnosti, 2005 cis-regulatory logic of short-range transcriptional repression in Drosophila melanogaster. Mol. Cell. Biol. 25: 34113420.

Kvon, E. Z., O. K. Kamneva, U. S. Melo, I. Barozzi, M. Osterwalder et al., 2016 Progressive Loss of Function in a Limb Enhancer during Snake Evolution. Cell 167: 633-642.e11.

Kwasnieski, J. C., C. Fiore, H. G. Chaudhari, and B. A. Cohen, 2014 High-throughput functional testing of ENCODE segmentation predictions. Genome Res. 24: 15951602.

Li, X.-Y., and M. B. Eisen, 2018 Mutation of sequences flanking and separating transcription factor binding sites in a Drosophila enhancer significantly alter its output. bioRxiv 379974.

Lim, B., T. Fukaya, T. Heist, and M. Levine, 2018 Temporal dynamics of pair-rule stripes in living Drosophila embryos. Proc. Natl. Acad. Sci. U. S. A. 115: 83768381.

Li, X.-Y., S. Thomas, P. J. Sabo, M. B. Eisen, J. A. Stamatoyannopoulos et al., 2011 The role of chromatin accessibility in directing the widespread, overlapping patterns of Drosophila transcription factor binding. Genome Biol. 12: R34.

Lopes, R., G. Korkmaz, and R. Agami, 2016 Applying CRISPR-Cas9 tools to identify and characterize transcriptional enhancers. Nat. Rev. Mol. Cell Biol. 17: 597-604. 
Ludwig, M. Z., Manu, R. Kittler, K. P. White, and M. Kreitman, 2011 Consequences of eukaryotic enhancer architecture for gene expression dynamics, development, and fitness. PLoS Genet. 7: e1002364.

Ludwig, M. Z., N. H. Patel, and M. Kreitman, 1998 Functional analysis of eve stripe 2 enhancer evolution in Drosophila: rules governing conservation and change. Development 125: 949-958.

Ma, L., J. Merenmies, and L. F. Parada, 2000 Molecular characterization of the TrkA/NGF receptor minimal enhancer reveals regulation by multiple cis elements to drive embryonic neuron expression. Development 127: 3777-3788.

Melnikov, A., A. Murugan, X. Zhang, T. Tesileanu, L. Wang et al., 2012 Systematic dissection and optimization of inducible enhancers in human cells using a massively parallel reporter assay. Nat. Biotechnol. 30: 271-277.

Milewski, R. C., N. C. Chi, J. Li, C. Brown, M. M. Lu et al., 2004 Identification of minimal enhancer elements sufficient for Pax3 expression in neural crest and implication of Tead2 as a regulator of Pax3. Development 131: 829-837.

Monti, R., I. Barozzi, M. Osterwalder, E. Lee, M. Kato et al., 2017 Limb-Enhancer Genie: An accessible resource of accurate enhancer predictions in the developing limb. PLoS Comput. Biol. 13: e1005720.

Ney, P. A., B. P. Sorrentino, K. T. McDonagh, and A. W. Nienhuis, 1990 Tandem AP-1binding sites within the human beta-globin dominant control region function as an inducible enhancer in erythroid cells. Genes Dev. 4: 993-1006. 
Noyes, M. B., X. Meng, A. Wakabayashi, S. Sinha, M. H. Brodsky et al., 2008 A systematic characterization of factors that regulate Drosophila segmentation via a bacterial one-hybrid system. Nucleic Acids Res. 36: 2547-2560.

Patwardhan, R. P., C. Lee, O. Litvin, D. L. Young, D. Pe'er et al., 2009 High-resolution analysis of DNA regulatory elements by synthetic saturation mutagenesis. Nat. Biotechnol. 27: 1173-1175.

Rogers, W. A., Y. Goyal, K. Yamaya, S. Y. Shvartsman, and M. S. Levine, 2017 Uncoupling neurogenic gene networks in the Drosophila embryo. Genes Dev. 31: 634-638.

Rohs, R., X. Jin, S. M. West, R. Joshi, B. Honig et al., 2010 Origins of specificity in protein-DNA recognition. Annu. Rev. Biochem. 79: 233-269.

Sabarís, G., I. Laiker, E. Preger-Ben Noon, and N. Frankel, 2019 Actors with Multiple Roles: Pleiotropic Enhancers and the Paradigm of Enhancer Modularity. Trends Genet. 35: 423-433.

Schroeder, M. D., C. Greer, and U. Gaul, 2011 How to make stripes: deciphering the transition from non-periodic to periodic patterns in Drosophila segmentation. Development 138: 3067-3078.

Shlyueva, D., G. Stampfel, and A. Stark, 2014 Transcriptional enhancers: from properties to genome-wide predictions. Nat. Rev. Genet. 15: 272-286.

Small, S., A. Blair, and M. Levine, 1992 Regulation of even-skipped stripe 2 in the Drosophila embryo. EMBO J. 11: 4047-4057.

Spitz, F., and E. E. M. Furlong, 2012 Transcription factors: from enhancer binding to developmental control. Nat. Rev. Genet. 13: 613-626. 
Staller, M. V., C. C. Fowlkes, M. D. J. Bragdon, Z. Wunderlich, J. Estrada et al., 2015 A gene expression atlas of a bicoid-depleted Drosophila embryo reveals early canalization of cell fate. Development 142: 587-596.

Vincent, B. J., M. V. Staller, F. Lopez-Rivera, M. D. J. Bragdon, E. C. G. Pym et al., 2018 Hunchback is counter-repressed to regulate even-skipped stripe 2 expression in Drosophila embryos. PLoS Genet. 14: e1007644.

White, M. A., 2015 Understanding how cis-regulatory function is encoded in DNA sequence using massively parallel reporter assays and designed sequences. Genomics 106: 165-170.

Wunderlich, Z., M. D. Bragdon, and A. H. DePace, 2014 Comparing mRNA levels using in situ hybridization of a target gene and co-stain. Methods 68: 233-241.

Zhou, H. Y., Y. Katsman, N. K. Dhaliwal, S. Davidson, N. N. Macpherson et al., 2014 A Sox2 distal enhancer cluster regulates embryonic stem cell differentiation potential. Genes Dev. 28: 2699-2711. 
bioRxiv preprint doi: https://doi.org/10.1101/2020.06.19.162164; this version posted June 20, 2020. The copyright holder for this preprint which was not certified Bypeer review) is the author/funder, who has granted bioRxiv a license to display the preprint in perpetuity. It is made

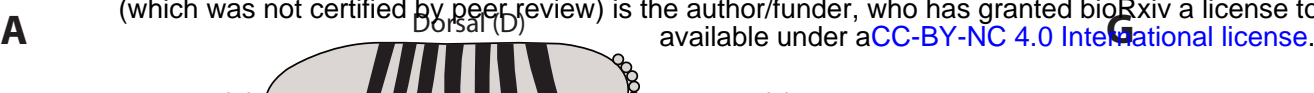

Anterior (A)

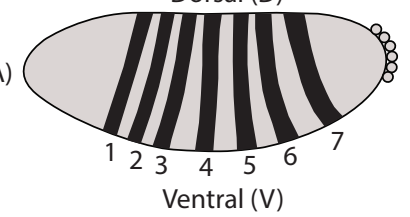

Posterior (P)

$3+7$

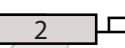

eve

\section{B}

$\operatorname{minWT}(484 \mathrm{bp})$

$\longrightarrow$ lacZ

extWT (798 bp)

$\operatorname{lacZ}$

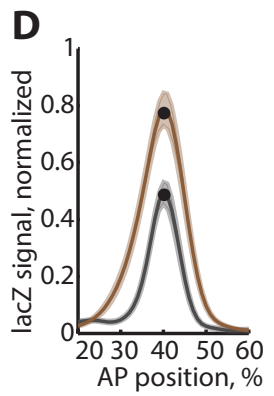

C

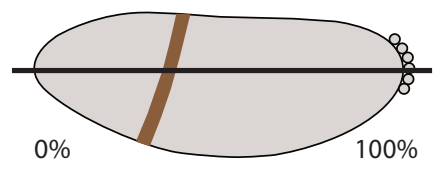

E $\quad$ ratio $>1=$

F

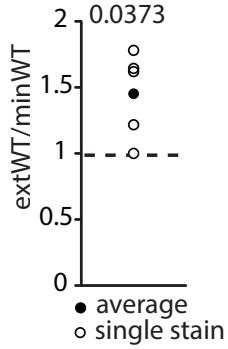

$4+6$

H
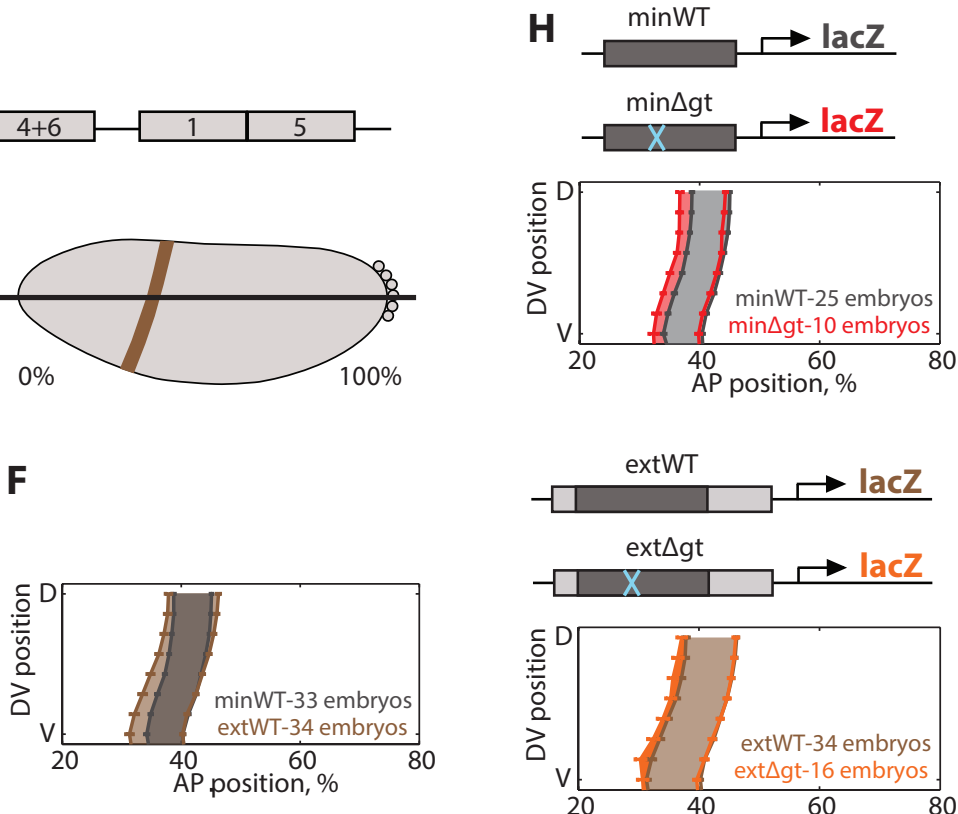

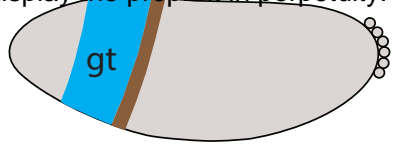

I
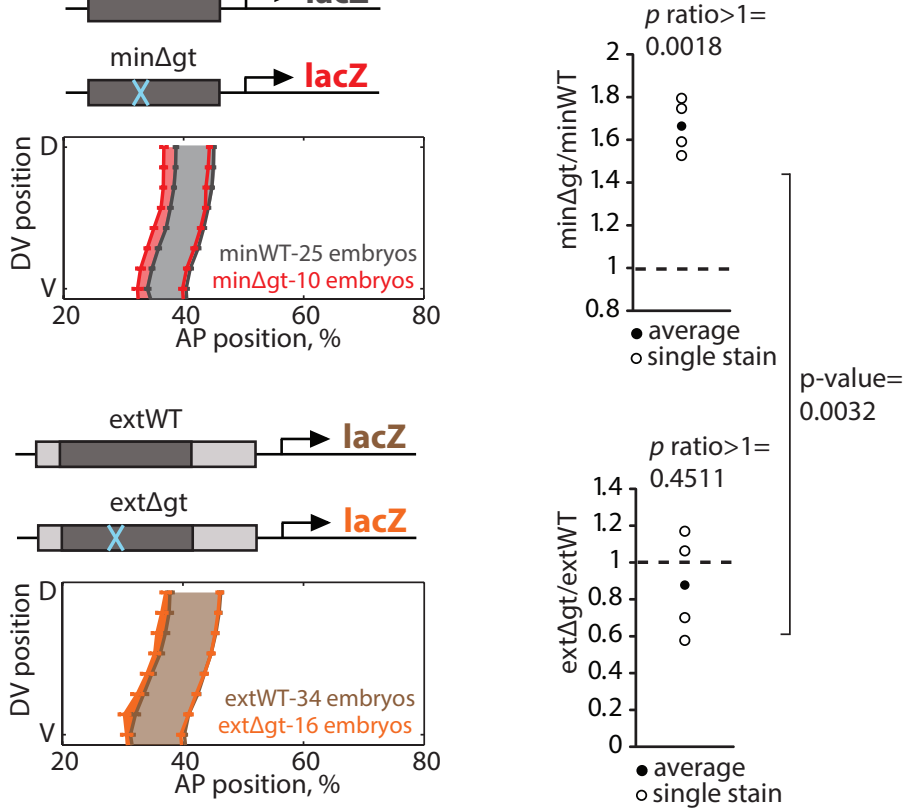
bioRxiv preprint doi: https://doi.org/10.1101/2020.06.19.162164; this version posted June 20, 2020. The copyright holder for this preprint A (which was not certified by peg review) is the author/funder, who has granted poRxiv a license to display the preprint in perpetuity. It is made
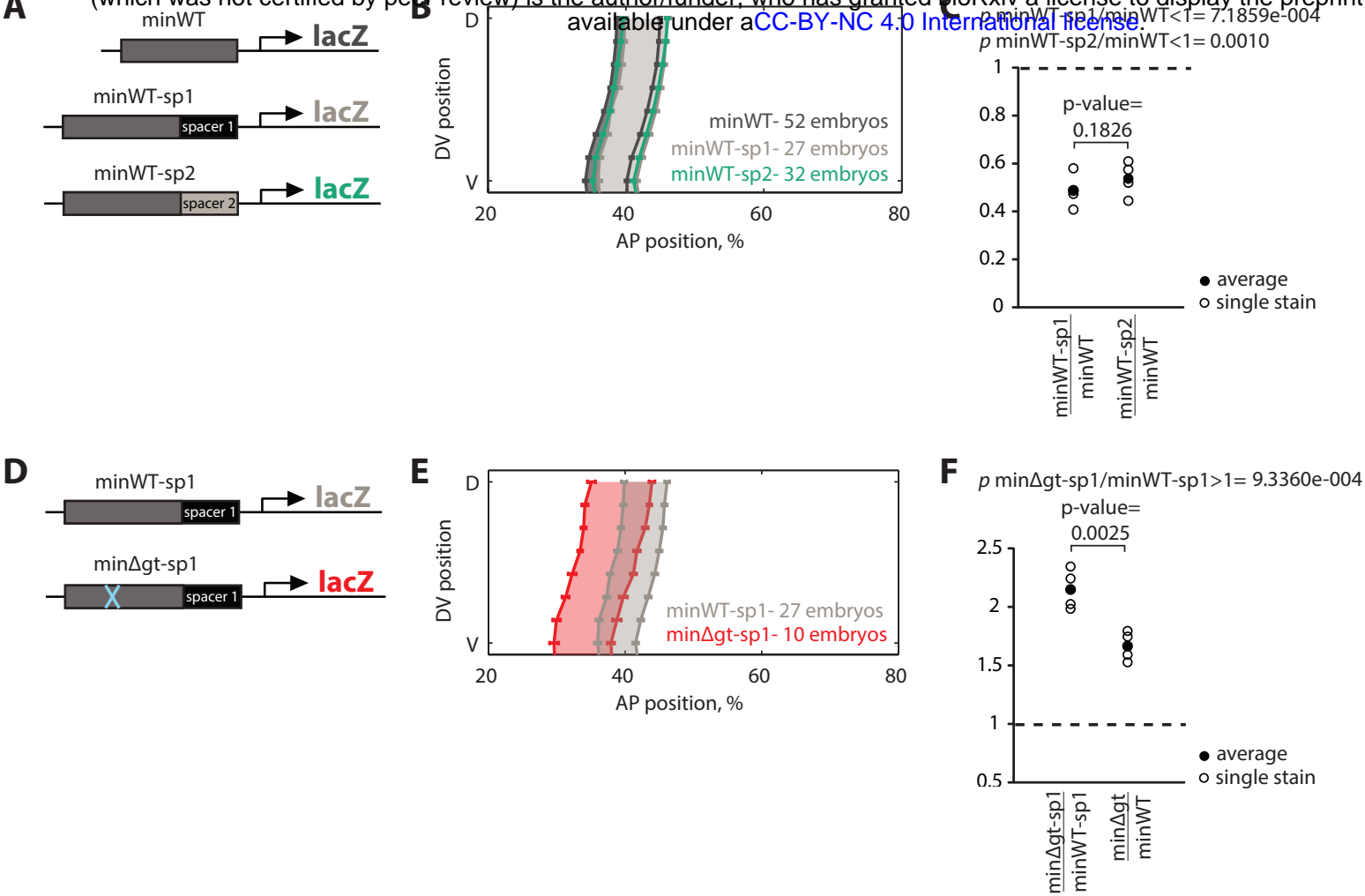
bioRxiv preprint doi: https://doi.org/10.1101/2020.06.19.162164; this version posted June 20, 2020. The copyright holder for this preprint

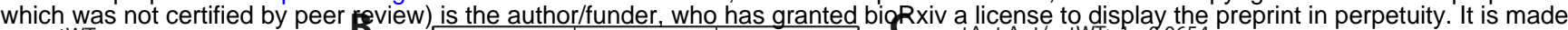
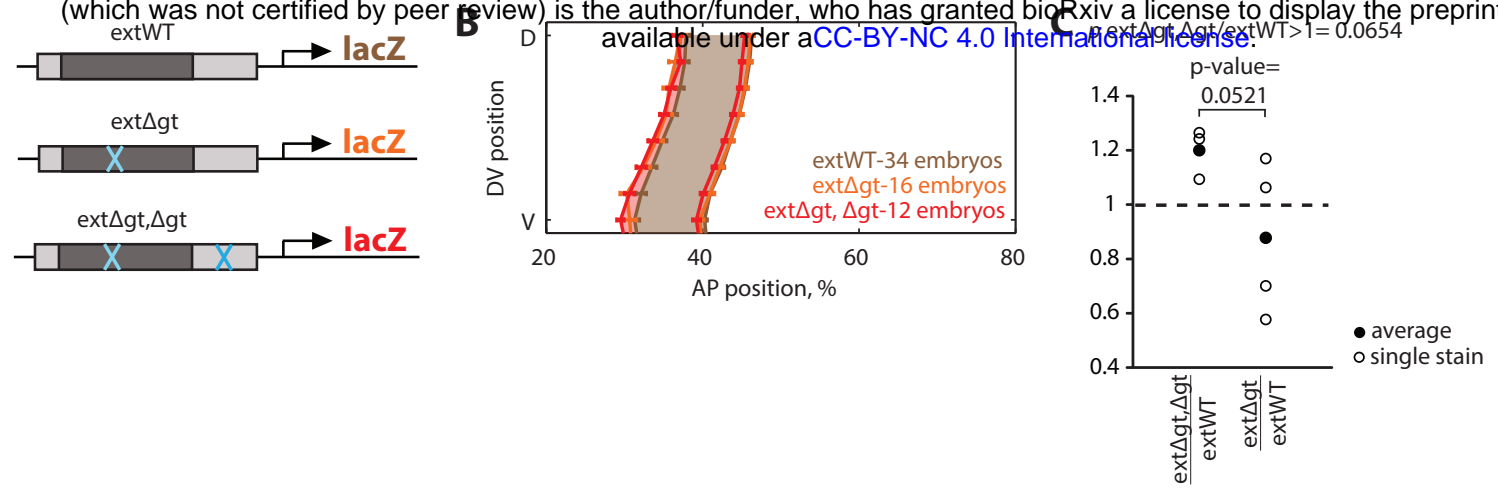

D

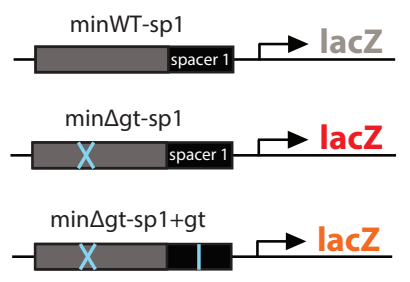

E

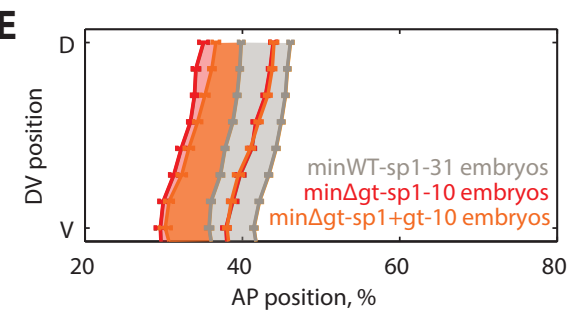

$\mathbf{F}$

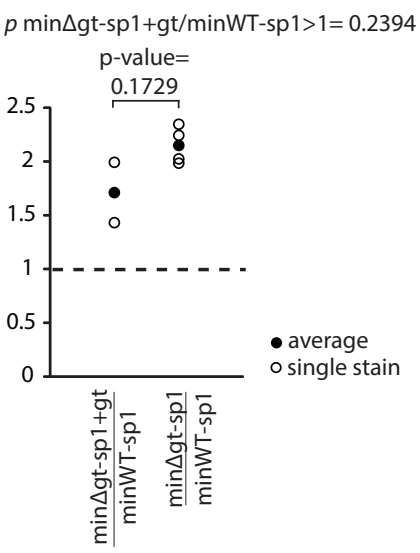


bioRxiv preprint doi: https://doi.org/10.1101/2020.06.19.162164; this version posted June 20, 2020. The copyright holder for this preprint (which was not certified by peer review) is the author/funder, who has granted bioRxiv a license to display the preprint in perpetuity. It is made available under aCC-BY-NC 4.0 International license.

A WT eve locus

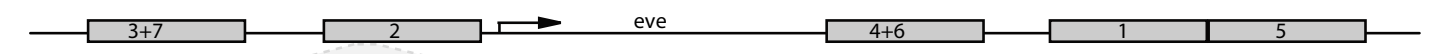

tattgcagcatcttgaacaatcgtcgcagtttggtaacacg

deltaGT eve locus
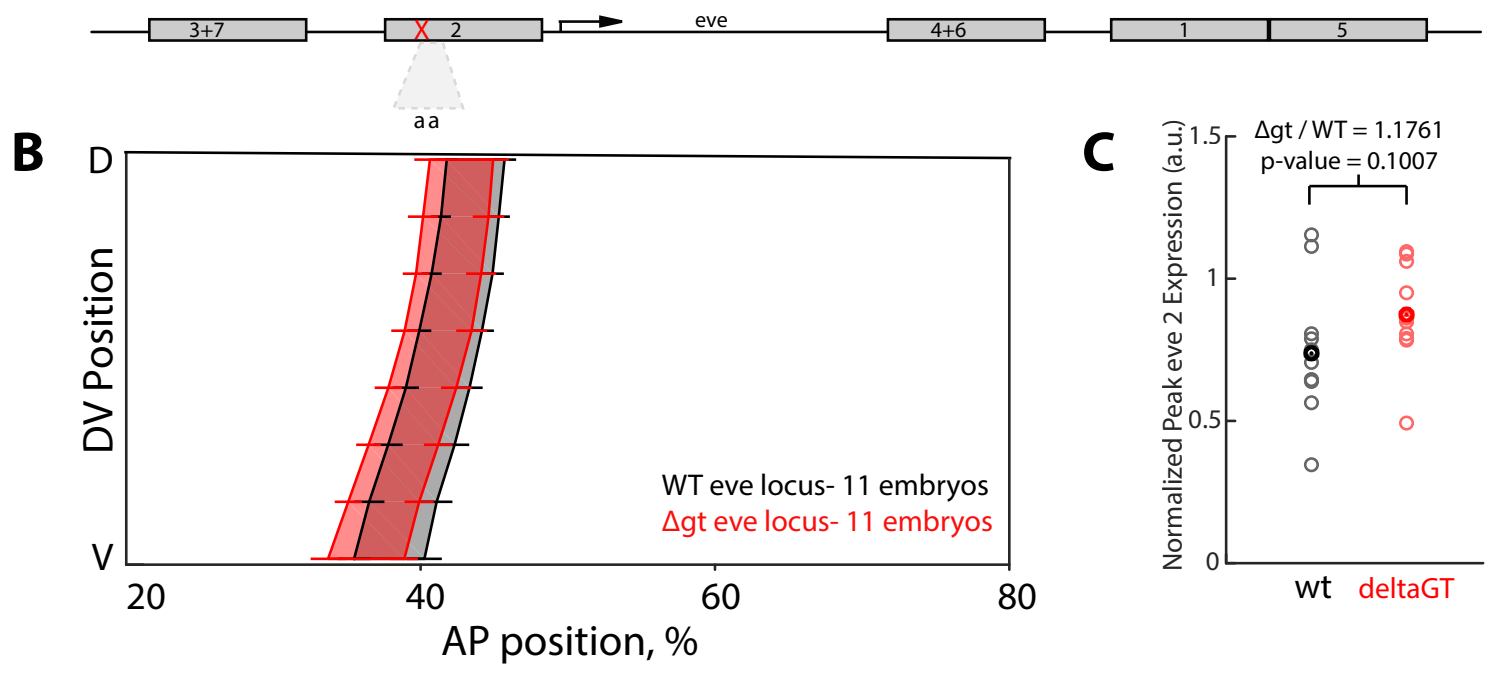

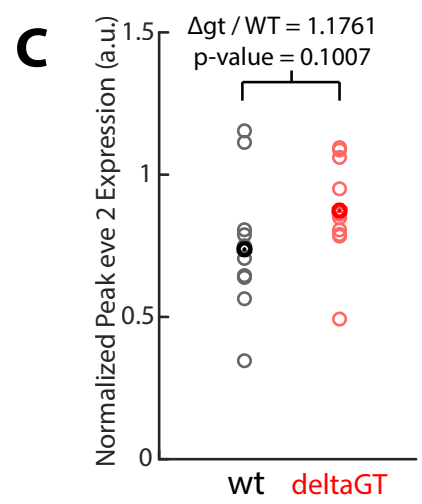

AP position, $\%$ 